\title{
Bearing/Incipient/Open Phase Fault Detection and Diagnosis of Multi-Phase Induction Motor Drives Equipped By GBDTI2HO Technique
}

\author{
Balamurugan Annamalai ${ }^{1}$, Sivakumaran Thangavel Swaminathan ${ }^{2}$ \\ ${ }^{1}$ Dept. of EEE, Sathyabama Institute of Science and Technology, Tamil Nadu, India. \\ ${ }^{2}$ Dept. of EEE, Sasurie College of Engineering, Tirupur, Tamil Nadu, India. \\ E-mail: *abalamurugan1205@gmail.com, tssivakumaran001@gmail.com
}

Received December 13, 2020; Revised January 14, 2021; Accepted February 20, 2021

\begin{abstract}
In this paper, a hybrid system is performed with fault detection and diagnosis on multi-phase induction motor (IM). The proposed method is hybrid of integrated Harris Hawk optimization (IHHO) and gradient boosting decision trees (GBDT) thus called the GBDTI2HO method. Here, additional operators are included in this paper to improve HHO's search behaviour namely crossover and mutation. Distorted waveforms are generated by different frequency patterns to indicate the time domain frequency as an assessment of failure. For this signal representation, the discrete wavelet transformation (DWT) is suggested. It extracts the characteristics and forwards them to IHHO technique to form the possible data sets. After the generation of the data set, GBDT classifies the ways of failure reached as winding of stator in multi-phase IM. The implementation of the proposed system is compared with existing systems, such as ANN, STransform and GBDT. The proposed method is executed on MATLAB/Simulink work platform to demonstrate the successfulness of proposed system, statistical measures are determined, as precision, sensitivity and specificity, mean median and standard deviation. For demonstrating the successfulness of proposed system, statistical measures are determined as precision, sensitivity, specificity, mean median as well as standard deviation. In 50 trails the proposed method, 0.98 for accuracy, 0.96 for specificity, 1.60 for recall as well as 0.97 for precision. In 100 trail the proposed method, 0.96 for accuracy, 0.93 for specificity, 0.87 for recall as well as 0.99 for precision.
\end{abstract}

Keywords: Multi-phase induction motor, Fault, Distorted waveforms, Frequency components, Stator winding, Statistical measures

\section{INTRODUCTION}

Three-phase engines should suggest the economy measures and technology that have been shown to control the speed in market drive use. However, the current initiatives have taken advantages of different phase machines with 
high point, and also get key methods that can be completed to three phase standards [1]. Fault tolerance is therefore implemented in various phases of redundancy in single-character machines, with industry initially focused on maximal reliability system as aerospace, traction or wind energy, with different stages of twisting machines on three stages. The electrical machine fault diagnosis is used to observe as rising failures, and correction leads to shorter idle hours and faster unplanned preservation to minimize harmful side effects. More kinds of failure (for instance broken rotor bars or failure among turns, to name a few) should be assumed and more detection time may be permitted. Although multiphase units have some methods for fault detection in bibliography they are mainly based on discrete wavelet transform or hilbert-huang transform, but no one meet the goal $[2,3]$.

\section{RELATED WORKS}

Various research methods in the literature that rely on multi-phase induction machine fault diagnosis used as several methods and features. Some of them are reviewed here.

Contreras-Hernandez et al. [4] has suggested an innovative engine fault detection system due to evaluation of signal as quaternion. Singh and Shaik [5] have employed the allocation, detection and location of defective bearings as IM on three-phase use from support vector machine and Stock-well transformer. Shao et al. [6] has suggested a deep studying due to various signals, because the failure diagnosis technique harnesses powerful studying capacity of convolution neural network (CNN). Failure diagnostic technique due to machine learning was suggested to Ali et al. [7] in induction motor as experimental data. Several unique and multi-electrical/ mechanical failures were performed on induction dual motor similar to the laboratory test. Hajary et al. [8] have presented a new fault diagnosis strategy in open phase is operated as three-phase of induction motor driven among entire speed range at various methods of burden.

Analysis of the current investigation shows a significant contribution to the observation of IM failures. More quantities of electrical units are installed as commercial plant. Therefore, the detection of health in individual engines is critical methods. Observing various failure modes as network neural, generalized likelihood ratio test (GLRT), fast Fourier transformation (FFT) so on. The artificial neural network (ANN) has the potential to use the impact of motor monitoring and observation as an economic, reliable and non-invasive system. Therefore, it cannot be executed as reasoning for heuristic failure observation systems. Consequently the major drawback of AI system is that the black box data structure methods and their maximal computation cost are done in real world applications. In addition, there is a problem with the first training stage, but require a maximal set of stator current databases for several state systems. This method is crucial as operation of optimal and misleads or outcomes obtain as restricted system set. Therefore, the impact of FFT assessment as another frequency domain method is required, as the 
rolling system frequency is known as pre-estimated. Another weakness is the increased trouble of spectrum classification since the signal-to-noise ratio is minimal and the spectrum as vibration has maximal frequency components depending on complexity of system. Although previous methods are used to predict failures among shifts, the complexity of algorithm is increased interms of largest number of samples required. To overwhelm such systems, it is necessary to observe the optimal use of the promoted method.

\section{Objectives and Contribution}

[a] In the proposed study, a hybrid system is performed interms of fault detection and diagnosis on multi-phase IM. The proposed method is hybridization of integrated Harris Hawk optimization (IHHO) and gradient boosting decision trees (GBDT) thus called the GBDTI2HO method. First, the analysis of faults like bearing, incipient and open phase fault is explained in multiphase drives. The feature extraction, dataset generation and fault classification is described. By then the performance efficiency of the proposed detection and classification of multiphase induction motor faults is performed on matrix laboratory/Simulink working platform and implementation is evaluated with existing systems.

[b] In traditional Harris Hawk Optimization (HHO), the slowest rate of prey search and premature convergence are the limitations. Therefore, additional operators are included in this paper to improve the HHOs search behavior namely crossover and mutation.

(c) There are three main functions in the proposed study to solve the optimization issue. The initial function of the proposed system is performed using discrete wavelet transform (DWT). In the proposed work, DWT is utilized to extract features and the extracted features are formed as a dataset to detect and classify failure due to frequencies using the GBDTI2HO technique.

(d) The second function of the proposed system is performed using Integrated Harris Hawk Optimization (IHHO). Here, the extracted features from DWT are fed to the IHHO technique to collect the possible datasets. The collected dataset is generated to classify the faults in the multiphase IM.

(e) The third function of the proposed system is performed through Gradient Boosting Decision Trees (GBDT). Here, GBDT is used to allocate the fault of the multiphase IM. Hence, ordinary and unordinary data are assessed at the beginning to demonstrate its unity. GBDT input as stator winding current.

Remaining manuscript is organized as below: Session 2 delineates literature survey and its background. Section 3 explains analysis of faults on multiphase induction motor (IM) drives. Section 4 delineates multiphase IM is evaluated utilize as proposed hybrid method. Section 5 describes the outcomes get with proposed and existing techniques. Session 6 concludes the manuscript. 


\section{ORIGINALITY}

In this section, the fault evaluation in multi-phase induction motor (IM) is explained for this section. In the field of electric drive design, the problems shows above are the most challenging factors [9].

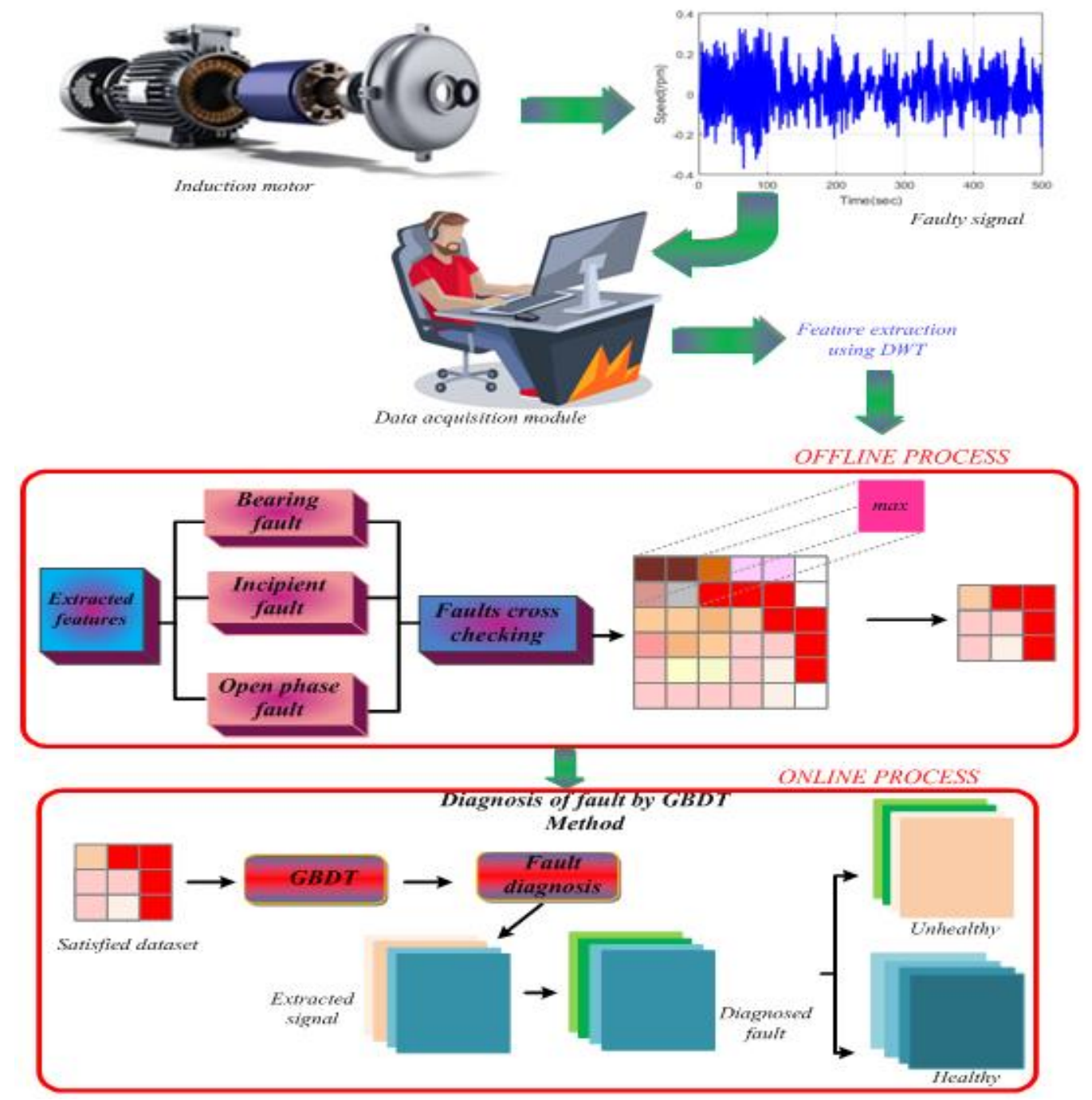

Figure 1: Proposed schematic for fault detection and classification on multiphase IM drives

For continuous operation, various phase drives does not require additional apparatus as superior operation of failure control. This requires only an appropriate superior scheme of failure control. Therefore, multiphase drives are optimal for security reasons i.e. traction and aerospace applications [10]. Fig 1 delineates the proposed observation scheme and assigns faults to IM in multi-phase drives. The main allocation of general failures in electric drives is formulated in the reference [11-16]. 


\section{SYSTEM DESIGN}

In this method, the multiphase evaluation of IM proposed hybrid methods. The proposed model is detained in next session.

\subsection{Feature Extraction Using Discrete Wavelet Transform (DWT)}

Discrete wavelet transform (DWT), in extract, refers to adjustment of linear works data as vector length becomes equal to the numerical vector of diverse length, since the power is two integers [17]. In this document, DWT is utilized to extract the characteristics and extracted characteristics are formed as a set of data to detect and classify failure due to frequencies using the GBDTI2HO technique. Hence, original transient current and multi-phase IM failure signals are broken down into a matrix of two coefficients namely approximate matrix and the detail coefficients. Here, the matrix of approximation coefficients such as cA, the matrix of detail coefficients such as $\mathrm{cH}, \mathrm{cV}$ and $\mathrm{cD}$ (horizontal, vertical and diagonal, respectively). The maximum component of the frequency signal is referred to as minimum-scale decomposition that represents the coefficients. The minimum component of frequency signals is referred to as large-scale decomposition called approximate coefficients. Thus, signals must be normalized to decrease the difficulty based on current magnitudes. In course of operation is stable state of multi-phase IM, current winding stator is normalized to evaluated value of peak. The most normalization factor is characterized by its competence to evaluate the multiphase IM failures of different evaluated values.

\subsection{Dataset Generation Using Integrated Harris Hawk Optimization (IHHO)}

In this section, the extracted features from DWT are fed to the IHHO technique to collect the possible datasets. The collected dataset is generated to classify the faults in the multiphase IM. In this article, a new population due to optimization paradigm, inspired by nature, known as Harris Hawks Optimization (HHO) [18, 19]. HHO search behaviour is enhanced by crossover and mutation operator.

Steps of IHHO

Step 1: Initialization: In this step, the initialization process and random generation is carried out. Here the input parameters such as stator winding current signals of multiphase IM.

Step 2: Evaluation: To utilize IHHO, four ways of data are collect as ordinary and plan cases, like $\mathrm{cA}, \mathrm{cH}, \mathrm{cV}$ and $\mathrm{cD}$. These outputs are used to analyze the fault type. If fault obtains in winding of multiphase IM stator, the failure character is also presented in wavelet coefficients of signals as current. When any condition of failure present in multiphase IM, it resends the coefficients of current wavelet for the fault allocation type. 
Step 3: Exploration Phase: To enhance as similar opportunity q as every hanger method, they perch due to the positions of family members other (to be close sufficient they attack) and rabbit.

Step 4: Exploitation Phase: In this phase, Harris's hawks carry out the surprise attack (seven murders as indicated) to find expected prey detect as previous phase.

Step 5: Crossover and Mutation: The solution can be improved by rearranging the Harris Hawks location using modes for consequent updating process using the crossover and mutation operator [20].

$$
\begin{gathered}
X=\frac{N_{G X}}{L_{c}} \\
M=\frac{M_{p}}{L_{c}}
\end{gathered}
$$

where $N_{G X}$ indicates the number of individuals crossover, $M_{p}$ denotes point of mutation as well as $L_{c}$ denotes prey length.

Step 6: Termination: Here, the best solution of the stator current signals are determined, in light of the fitness values and the network is prepared to give the possible dataset as indicated by multiphase IM fault. The algorithm of outer IHHO, which is programmed as the equations below, contains the data set to the minimum and maximum frequency as current stator.

$$
D S_{\text {IHHO }}=\left[\begin{array}{c}
c A^{1} \\
c A^{2} \\
\vdots \\
c A^{n}
\end{array}\right]^{c, v}\left[\begin{array}{c}
c H^{1} \\
c H^{2} \\
\vdots \\
c H^{n}
\end{array}\right]^{c, v}\left[\begin{array}{c}
c V^{1} \\
c V^{2} \\
\vdots \\
c V^{n}
\end{array}\right]^{c, v}\left[\begin{array}{c}
c D^{1} \\
c D^{2} \\
\vdots \\
c D^{n}
\end{array}\right]^{c, v}
$$

where $c A_{n}^{c, v}, c H_{n}^{c, v}, c V_{n}^{c, v}$ and $c D_{n}^{c, v}$ denotes as minimum \& maximum signal of current frequency. Datasets are provided for GBDT input. Figure 2 elucidates the flowchart of the proposed hybrid technique. 


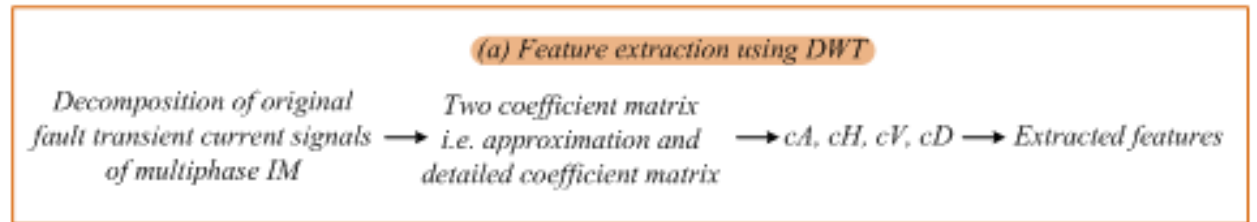

(b) Dataset generation using $\mathrm{HH} O$

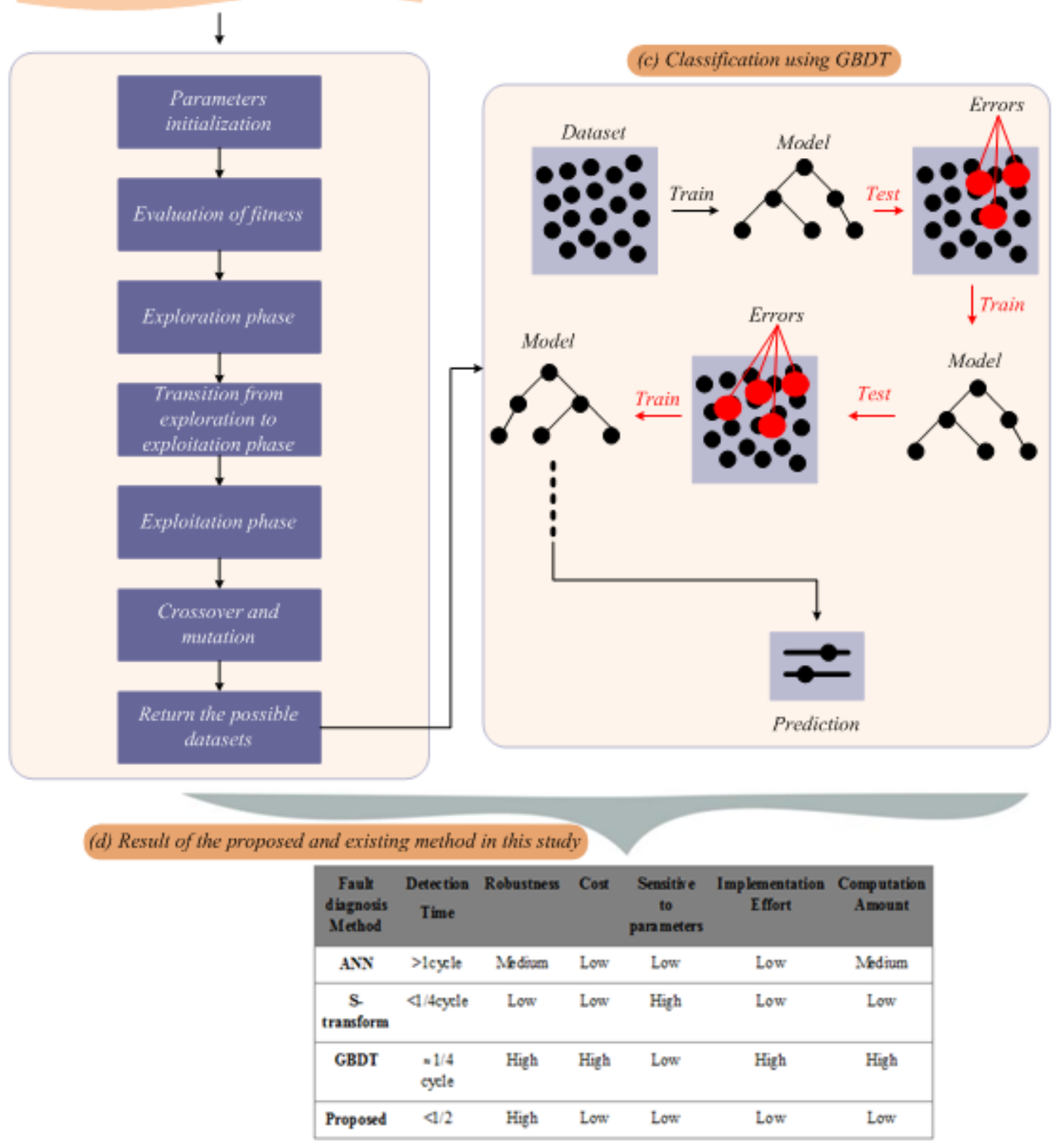

Figure 2: Flowchart and obtained outcomes of proposed hybrid technique 


\subsection{Fault Classification of Multiphase IM Using Gradient Boosting Decision Trees (GBDT)}

In this section, GBDT is used to allocate the fault of multiphase IM. Hence, ordinary and unordinary data are assessed at the beginning to demonstrate its unity. GBDT refers to internal winding current stator. Gradient augmentation utilize machine learning method as a reversal and allocation impact, which maintains a model of forecast form as a set of weak forecast techniques, decision tree as typical [21]. In this method, goal function is assessed due to computation of precision for every allocation. To compute the accuracy is,

$$
\operatorname{Accuracy}(\%)=\frac{T P+T N}{T P+F N+F P+T N} \times 100
$$

Here $T P$ and $T N$ represents the number of forecast correction for actual class as true and false. $F N$ and $F P$ represents number of forecast incorrect for actual class as true and false. Finally, the minimized value of the error is computed to obtain the inputs. Due to the behavior ability, GBDT is trained as optimal and obtain optimal results and classification related precision is calculated. The outcome of simulation of proposed hybrid method with the existing method analyzed at MATLAB/Simulink platform. They are comes under the following section.

\section{EXPERIMENT AND ANALYSIS}

This session, depicts the outcomes and conversation of fault detection and classification in multiphase IM. It contains three kinds of fault (1) bearing (2) incipient (3) open phase. Initially, Induction motor (IM) behaviors are analyzed under normal and faulty conditions. Then performance of current stator is normalized with peak value rate. Then, the performance of failure functions of signal in current is extracted as a Discrete Wave Transformation. Extracted characteristics are handled in an advanced manner to provide the failure types. The performances of the DWT system is assessed and compared to ANN, GDBT, S-Transform methods, and statistical measures like precision, sensitivity, specificity values also evaluated.

\subsection{Bearing Fault}

Figure 3 describes the DWT performance of multiphase IM speed under normal operation. Figure 4 shows the performance of multiphase IM speed under ball fault. Figure 5 shows the performance of multiphase IM speed on cantered fault. Figure 6 shows the performance of multiphase IM speed under inner race failure. 


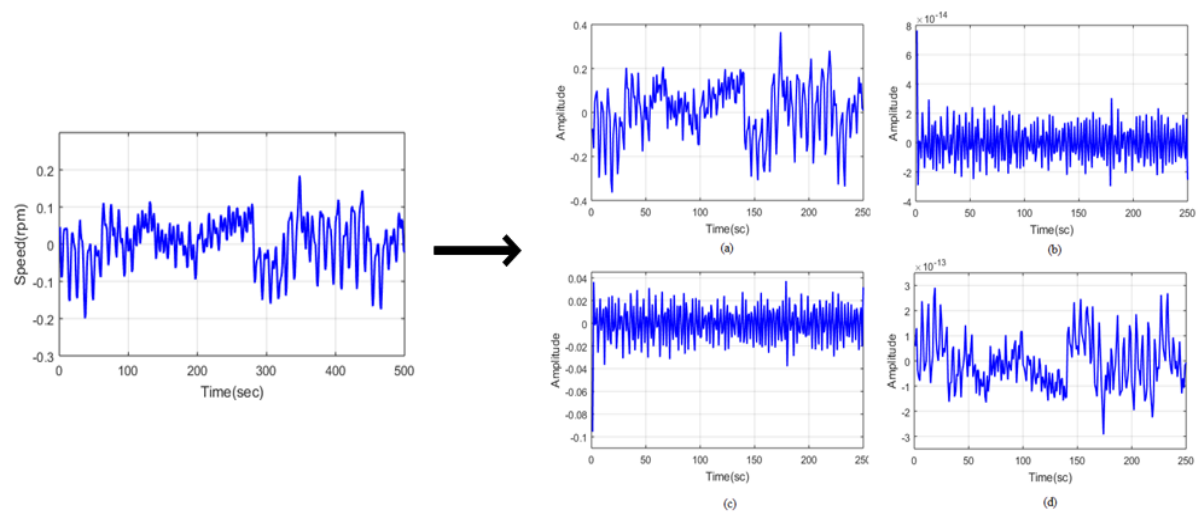

Figure 3: DWT Performance of Multiphase IM speed under normal operation (a) cA (b) cD (c) cH (d) cV

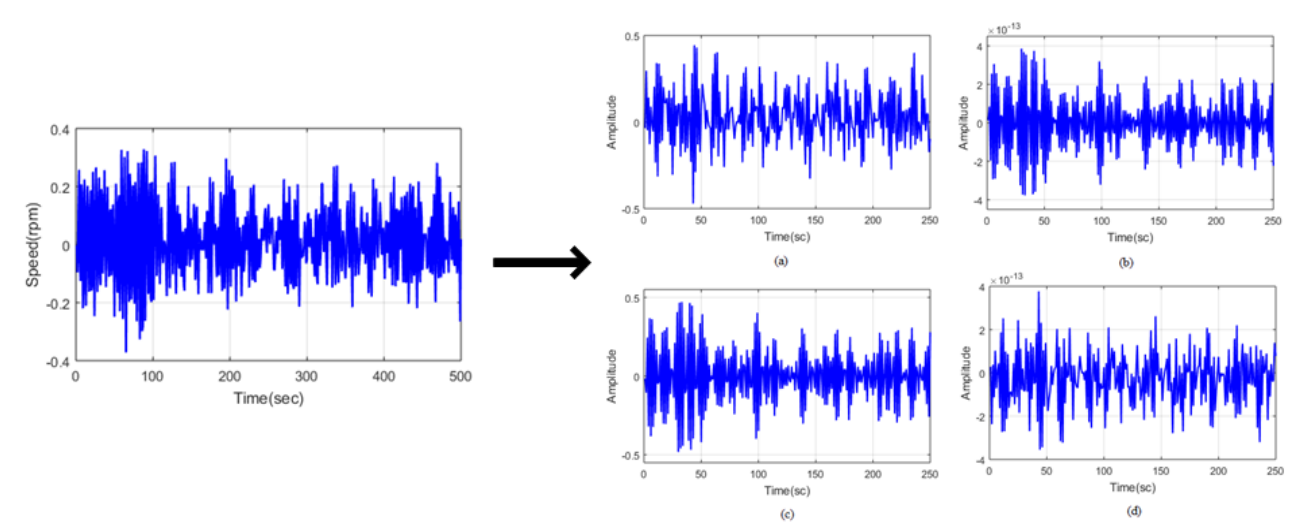

Figure 4: DWT Performance of Multiphase IM speed under ball fault (a) cA (b) $\mathrm{cD}(\mathrm{c}) \mathrm{cH}(\mathrm{d}) \mathrm{cV}$
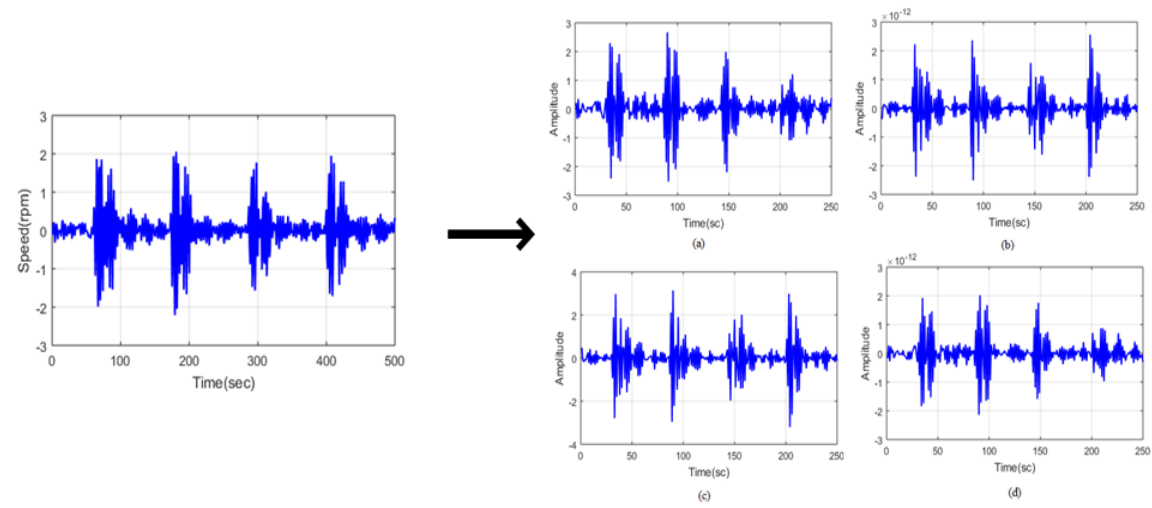

Figure 5: DWT performance of Multiphase IM speed under centred fault (a) $\mathrm{cA}(\mathrm{b}) \mathrm{cD}(\mathrm{c}) \mathrm{cH}(\mathrm{d}) \mathrm{cV}$ 

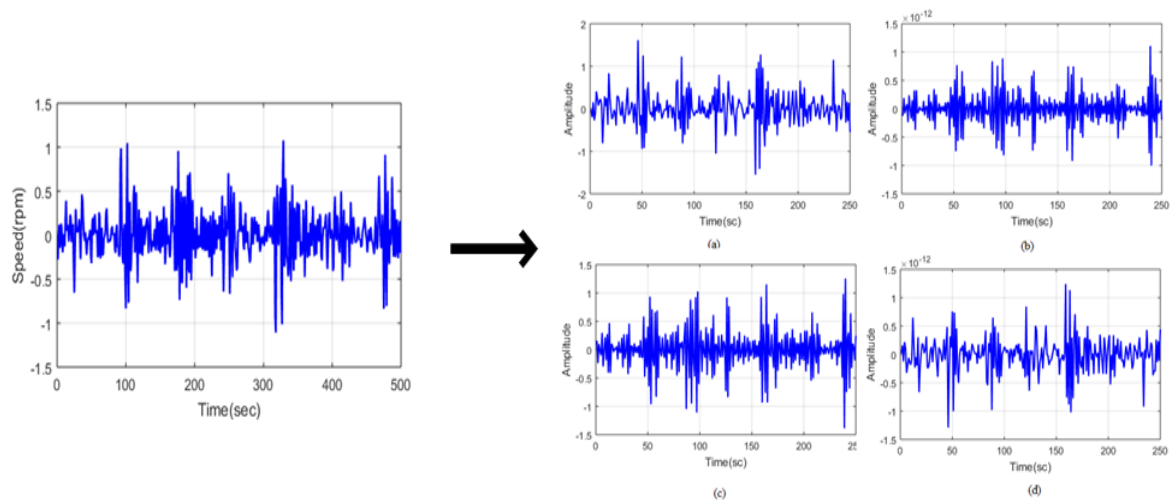

Figure 6: DWT performance of Multiphase IM speed under Inner race fault (a) $\mathrm{cA}(\mathrm{b}) \mathrm{cD}$ (c) $\mathrm{cH}$ (d) $\mathrm{cV}$

\subsection{Performance Analysis}

This session shows the performance of proposed GBDTI2HO method. Subject to classes true positive (TP), true negative (TN), false positive (FP) and false negative (FN), the yields of the assignment are suggested.

\subsection{Modeling Metrics Evaluation}

The modeling metrics are calculated for error computation on this section, as (1) Root Mean Square Error (RMSE), Mean Absolute Percentage Error (MAPE) and Mean Bias Error (MBE). Table 1 displays accuracy, sensitivity and specificity of GBDTI2HO technique.

Table 1: Accuracy, Sensitivity, and Specificity of GBDTI2HO technique

\begin{tabular}{ccccccccc}
\hline \multicolumn{2}{c}{$\begin{array}{c}\text { Types of } \\
\text { fault }\end{array}$} & TP & TN & FP & FN & Accuracy & Sensitivity & Specificity \\
\hline \multirow{4}{*}{ Bearing } & Ball & 2 & 14 & 1 & 1 & 0.95 & 0.63 & 0.90 \\
fault & Inner race & 2 & 15 & 1 & 1 & 0.94 & 0.68 & 0.89 \\
& Opposite & 3 & 11 & 0 & 1 & 0.95 & 0.64 & 0.89 \\
& Orthogonal & 2 & 12 & 0 & 2 & 0.95 & 0.65 & 0.89 \\
\hline \multirow{5}{*}{ Incipient } & $\mathrm{A}$ & 1 & 12 & 1 & 1 & 0.95 & 0.62 & 0.90 \\
fault & $\mathrm{B}$ & 2 & 13 & 0 & 2 & 0.95 & 0.68 & 0.90 \\
& $\mathrm{C}$ & 2 & 14 & 1 & 2 & 0.95 & 0.63 & 0.90 \\
& $\mathrm{AB}$ & 3 & 14 & 0 & 1 & 0.95 & 0.63 & 0.91 \\
& $\mathrm{BC}$ & 4 & 14 & 1 & 1 & 0.95 & 0.62 & 0.91 \\
Open & $\mathrm{AC}$ & 4 & 11 & 1 & 1 & 0.95 & 0.66 & 0.90 \\
phase & $\mathrm{ABC}$ & 3 & 11 & 1 & 2 & 0.95 & 0.66 & 0.89 \\
fault & $\mathrm{A}$ & 4 & 12 & 1 & 2 & 0.95 & 0.61 & 0.89 \\
Normal & $\mathrm{B}$ & 3 & 14 & 0 & 1 & 0.95 & 0.67 & 0.90 \\
& $\mathrm{C}$ & 1 & 11 & 1 & 2 & 0.94 & 0.63 & .91 \\
& Normal & 4 & 12 & 1 & 2 & 0.95 & 0.61 & 0.89 \\
\hline
\end{tabular}




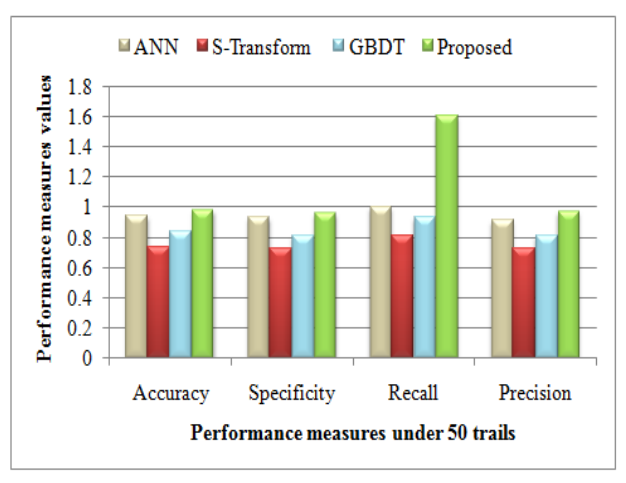

(a)

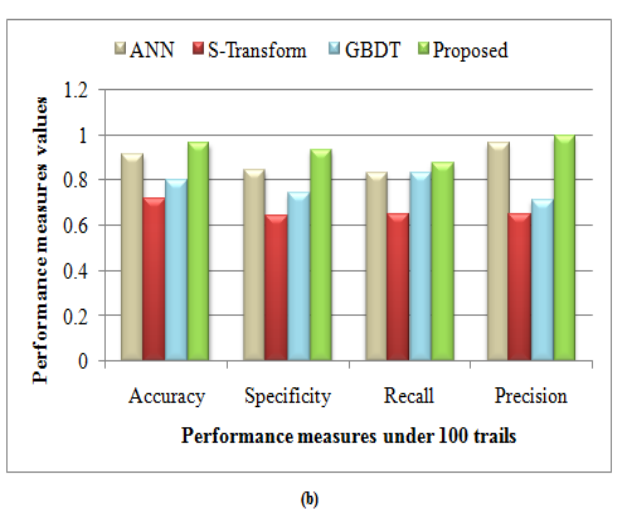

Figure 7: Analysis comparison of GBDTI2HO with existing technique in line signal (a) 50 (b) 100 number of trails

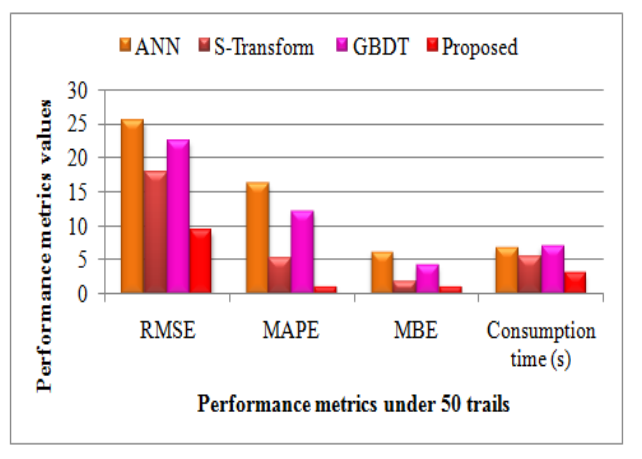

(a)

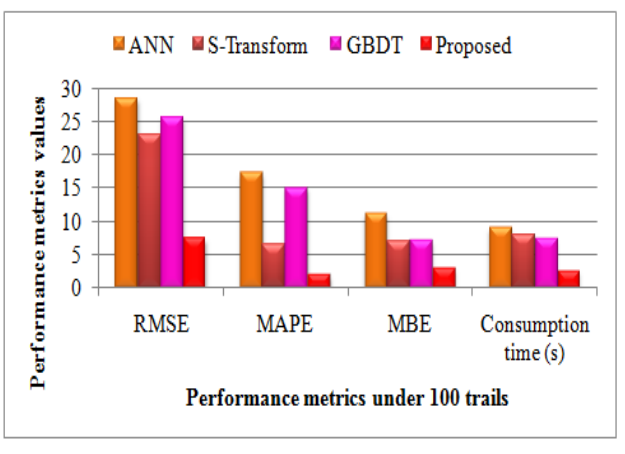

(b)

Figure 8: Modeling metrics of proposed to existing technique due to turn insulation failure of (a) 50 and (b) 100 trails number

Fig 7 shows the precision, specification, recall and precise of proposed and existing technique for 50 and 100 trails is presented. Figure 8 shows the MAPE, RMSE, MBE as well as time for consumption in proposed and existing technique.

Fig 9 displays the statistic evaluation of proposed and existing system. Table 2 shows the comparison of fault detection between proposed and existing method. Figure 10 (a) displays fitness of proposed method. Here the fitness is 1 in iteration 25. In Figure 10 (b) fitness comparison of proposed as well as existing method is presented. Compared to existing system fitness of GBDTI2HO system is optimal. In general, it clearly displays which proposed hybrid technique reach best outcome contrast to existing methods. 


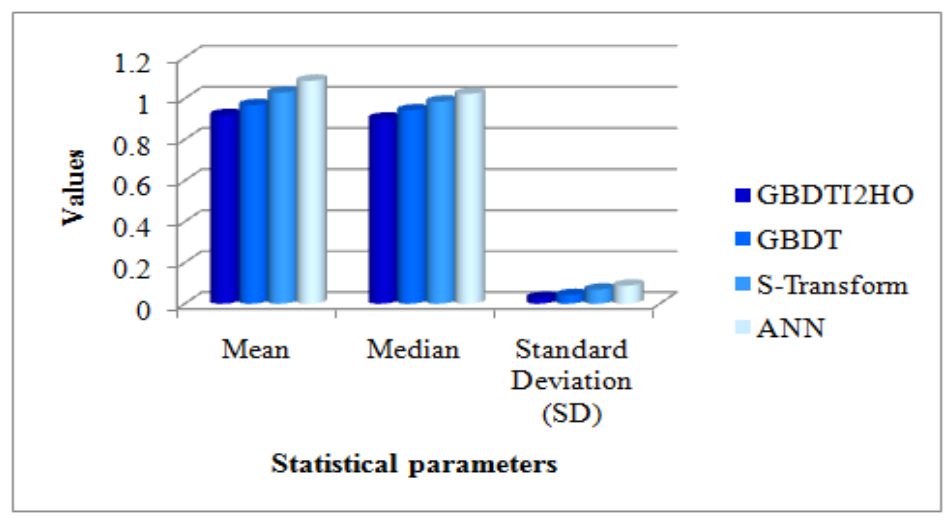

Figure 9: Statistic parameters of GBDTI2HO and existing systems

Table 2: Comparison of fault detection between proposed and existing methods

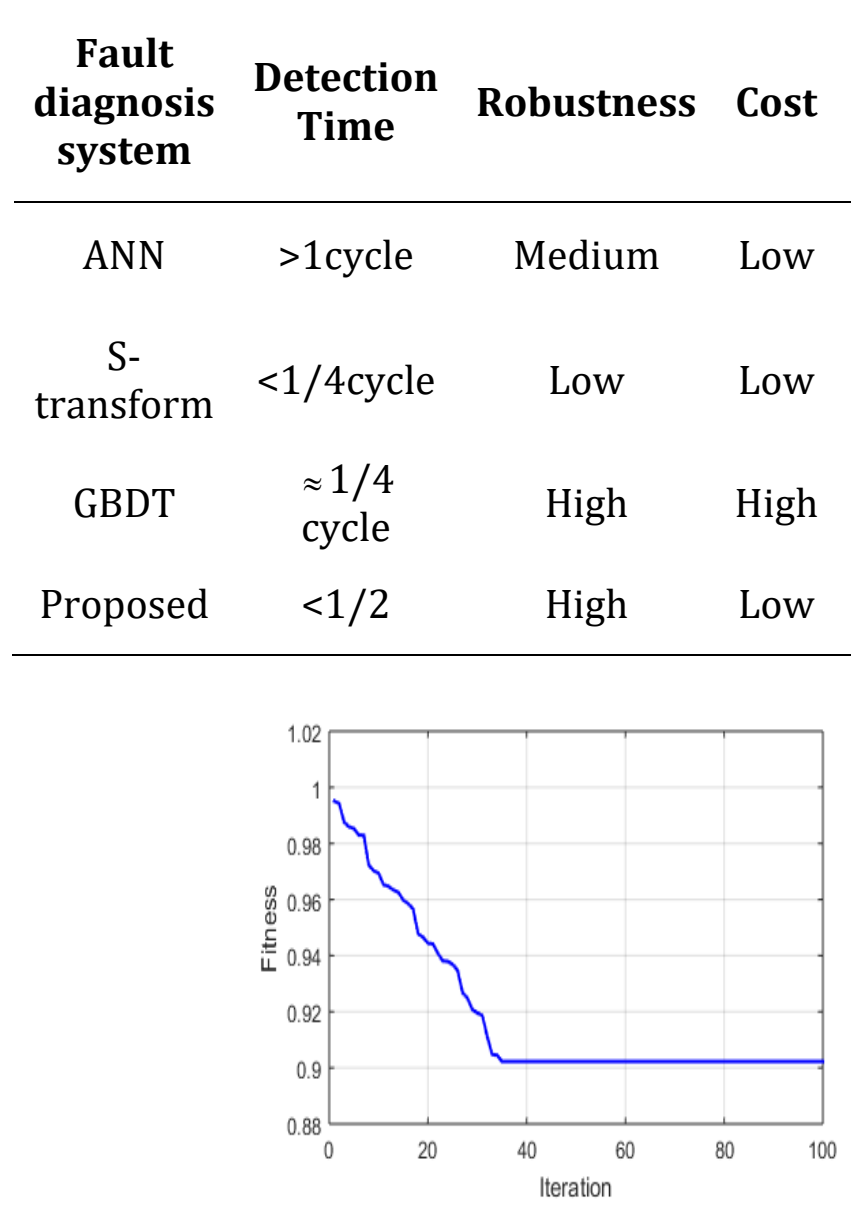

(a)

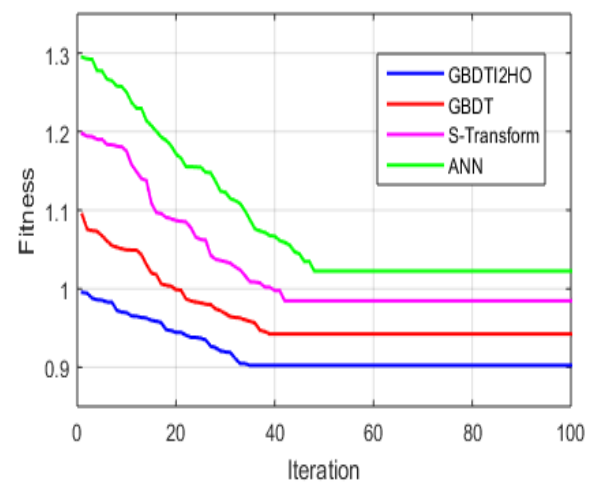

(b)

Figure 10: Fitness analysis (a) GBDTI2HO system (b) Comparison of GBDTI2HO and existing technique 


\section{CONCLUSION}

In this paper, GBDTI2HO system, fault detection and classification are examined in the multiphase IM. The fault signals are evaluated like DWT is provided for removing the characters from the interior signals as minimal and maximal frequency components. The IHHO employed for generating probable datasets. GBDT used for allocating the signals as failure or not. The proposed system for recognizing the path of failure is evaluated to MATLAB/Simulink platform. The success of GBDTI2HO system is associated. The efficiency of the GBDTI2HO method is verified using statistic measures like accuracy, sensitivity and specificity, in addition to S-Transform, ANN, and GBDT system. It is best by the existing system for determining the GBDTI2HO system interms of performance variant and statistical measure.

\section{REFERENCES}

[1] Elbouchikhi, Elhoussin, Yassine Amirat, Gilles Feld, and Mohamed Benbouzid. Generalized Likelihood Ratio Test Based Approach for Stator-Fault Detection in a PWM Inverter-Fed Induction Motor Drive. IEEE Transactions on Industrial Electronics. Vol. 66, No. 8, pp. 6343-6353, 2019. doi:10.1109/tie.2018.2875665

[2] Maraaba, Luqman, Zakariya Al-Hamouz, and Mohammad Abido. An Efficient Stator Inter-Turn Fault Diagnosis Tool for Induction Motors. Energies (Basel) Vol. 11, No. 3, pp. 653, 2018. doi:10.3390/en11030653

[3] Rebouças Filho, Pedro Pedrosa, Navar MM Nascimento, Igor R. Sousa, Cláudio MS Medeiros, and Victor Hugo C. de Albuquerque. A reliable approach for detection of incipient faults of short-circuits in induction generators using machine learning. Computers \& Electrical Engineering. Vol. 71, pp. 440-451, 2018. doi:10.1016/j.compeleceng.2018.07.046

[4] Contreras-Hernandez, Jose L., Dora Luz Almanza-Ojeda, Sergio Ledesma-Orozco, Arturo Garcia-Perez, Rene J. Romero-Troncoso, and Mario A. Ibarra-Manzano. Quaternion Signal Analysis Algorithm for Induction Motor Fault Detection. IEEE Transactions on Industrial Electronics. Vol. 66, No. 11, pp. 8843-8850, 2019. doi:10.1109/tie.2019.2891468

[5] Singh, Megha, and Abdul Gafoor Shaik. Faulty bearing detection, classification and location in a three-phase induction motor based on Stockwell transform and support vector machine. Measurement. Vol. 131, pp. 524-533, 2019. doi:10.1016/j.measurement.2018.09.013

[6] Shao, Siyu, Ruqiang Yan, Yadong Lu, Peng Wang, and Robert Gao. DCNNbased Multi-signal Induction Motor Fault Diagnosis. IEEE Trans Instrum Meas. pp. 1-1, 2019. doi:10.1109/tim.2019.2925247

[7] Ali, Mohammad Zawad, Md Nasmus Sakib Khan Shabbir, Xiaodong Liang, Yu Zhang, and Ting $\mathrm{Hu}$. Machine Learning-Based Fault Diagnosis for Single- and Multi-Faults in Induction Motors Using 
Measured Stator Currents and Vibration Signals. IEEE Trans Ind Appl. Vol. 55, No. 3, pp. 2378-2391, 2019. doi:10.1109/tia.2019.2895797

[8] Hajary, Ali, Reza Kianinezhad, S. Gh Seifossadat, S. S. Mortazavi, and Alireza Saffarian. Detection and Localization of Open-Phase Fault in Three-Phase Induction Motor Drives Using Second Order Rotational Park Transformation. IEEE Trans Power Electron. Vol. 34, No. 11, pp. 11241-11252, 2019. doi:10.1109/tpel.2019.2901598

[9] Consoli Alfio. Special Section on Robust Operation of Electrical Drives. IEEE Trans Power Electron. Vol. 27, No. 2, pp. 476-478, 2012. doi: 10.1109/tpel.2011.2173231

[10] de Lillo, Liliana, Lee Empringham, Pat W. Wheeler, Sudarat Khwan-On, Chris Gerada, M. Nazri Othman, and Xiaoyan Huang. Multiphase Power Converter Drive for Fault-Tolerant Machine Development in Aerospace Applications. IEEE Transactions on Industrial Electronics. Vol. 57, No. 2, pp. 575-583, 2010. doi:10.1109/tie.2009.2036026

[11] Gnanaprakasam C, Chitra K. S-transform and ANFIS for detecting and classifying the vibration signals of induction motor. Journal of Intelligent \& Fuzzy Systems. Vol. 29, No. 5, pp. 2073-2085, 2015. doi:10.3233/ifs-151684

[12] Hassan, Ola E., Motaz Amer, Ahmed K. Abdelsalam, and Barry Williams. Induction motor broken rotor bar fault detection techniques based on fault signature analysis - a review. IET Electric Power Applications. Vol. 12, No. 7, pp. 895-907, 2018. doi:10.1049/ietepa.2018.0054

[13] Surya Gulamfaruk, Khan Z, Makarand Ballal, Hiralal Suryawanshi. A Simplified Frequency-Domain Detection of Stator Turn Fault in Squirrel-Cage Induction Motors Using an Observer Coil Technique. IEEE Transactions on Industrial Electronics. Vol. 64, No. 2, pp. 1495-1506, 2017. doi:10.1109/tie.2016.2611585

[14] Wu, Yunkai, Bin Jiang, and Yulong Wang. Incipient winding fault detection and diagnosis for squirrel-cage induction motors $\begin{array}{lllll}\text { equipped on } & \text { CRH trains. ISA Trans. } & 2019 .\end{array}$ doi:10.1016/j.isatra.2019.09.020

[15] Yang, Shih-Chin, Yu-Liang Hsu, Po-Huan Chou, Guan-Ren Chen, and DaRen Jian. Online Open-Phase Fault Detection for Permanent Magnet Machines With Low Fault Harmonic Magnitudes. IEEE Transactions on Industrial Electronics. Vol. 65, No. 5, pp. 4039-4050, 2018. doi: 10.1109/tie.2017.2758752

[16] Kuruppu Sandun, Kulatunga N. D-Q Current Signature-Based Faulted Phase Localization for SM-PMAC Machine Drives. IEEE Transactions on Industrial Electronics. Vol. 62, No. 1, pp. 113-121, 2015. doi:10.1109/tie.2014.2334652

[17] Safari, Azadeh, Cheecottu Vayalil Niras, and Yinan Kong. Powerperformance enhancement of two-dimensional RNS-based DWT 
image processor using static voltage scaling. Integration. Vol. 53, pp. 145-156, 2016. doi:10.1016/j.vlsi.2015.12.006

[18] Transpire Online, (2020). An Efficient Harris Hawks Optimization (HHO) Algorithm for Solving Numerical Expressions, Transpire Online 2019. Available at:

https://transpireonline.blog/2020/01/28/an-efficient-harris-hawksoptimization-hho-algorithm-for-solving-numerical-expressions/.

[Accessed on: Mar, 2020]

[19] Heidari, Ali Asghar, Seyedali Mirjalili, Hossam Faris, Ibrahim Aljarah, Majdi Mafarja, and Huiling Chen. Harris hawks optimization: Algorithm and applications. Future Generation Computer Systems. Vol. 97, pp. 849-872, 2019. doi:10.1016/j.future.2019.02.028

[20] Kartci, Aslihan, Agamyrat Agambayev, Mohamed Farhat, Norbert Herencsar, Lubomir Brancik, Hakan Bagci, and Khaled N. Salama. Synthesis and Optimization of Fractional-Order Elements Using a Genetic Algorithm. IEEE Access, Vol. 7, pp. 80233-80246, 2019. doi: 10.1109/access.2019.2923166

[21] Rao, Haidi, Xianzhang Shi, and Ahoussou Kouassi Rodrigue. Feature selection based on artificial bee colony and gradient boosting decision tree.Appl Soft Comput, Vol. 74, pp. 634-642, 2019. doi:10.1016/j.asoc.2018.10.036 